\title{
The posets of classes of isomorphic subgroups of finite groups
}

\author{
Marius Tărnăuceanu \\ February 17, 2015
}

\begin{abstract}
In this paper we introduce and study the poset of equivalence classes of subgroups of a finite group $G$, induced by the isomorphism relation. This contains the well-known lattice of solitary subgroups of $G$. We prove that in several particular cases it determines the structure of $G$.
\end{abstract}

MSC (2010): Primary 06A06, 20D30; Secondary 06B99, 20D99.

Key words: isomorphic subgroups, subgroup lattices, posets, lattices, poset/lattice isomorphisms.

\section{Introduction}

The relation between the structure of a group and the structure of its lattice of subgroups constitutes an important domain of research in group theory. The topic has enjoyed a rapid development starting with the first half of the 20th century. Many classes of groups determined by different properties of partially ordered subsets of their subgroups (especially lattices of subgroups) have been identified. We refer to Suzuki's book [12], Schmidt's book [11] or the more recent book [14] by the author for more information about this theory.

It is an usual technique to consider an equivalence relation $\sim$ on an algebraic structure and then to study the factor set with respect to $\sim$, partially ordered by certain ordering relations. In the case of subgroup lattices, one of 
the most significant example is the poset $C(G)$ of conjugacy classes of subgroups of a group $G$ (see [2, 3, 4] and [9, 10]). The current paper deals with the more general equivalence relation on the subgroup lattice of $G$ induced by isomorphism. It leads to the set $\operatorname{Iso}(G)$ consisting of all equivalence classes of isomorphic subgroups of $G$, that becomes a poset under a suitable ordering relation. Its detailed study is the main goal of our paper. We investigate the finite groups $G$ for which the corresponding poset $\operatorname{Iso}(G)$ is a lattice and, in particular, a chain. We also give some information about the finite groups $G_{1}$ and $G_{2}$ for which the posets $\operatorname{Iso}\left(G_{1}\right)$ and $\operatorname{Iso}\left(G_{2}\right)$ are isomorphic.

In the following for a finite group $G$ we will denote by $L(G)$ the subgroup lattice of $G$. Recall that $L(G)$ is a complete bounded lattice with respect to set inclusion, having initial element the trivial subgroup 1 and final element $G$, and its binary operations $\wedge, \vee$ are defined by

$$
H \wedge K=H \cap K, H \vee K=\langle H \cup K\rangle \text {, for all } H, K \in L(G) \text {. }
$$

Two groups $G_{1}$ and $G_{2}$ will be called L-isomorphic if their subgroup lattices $L\left(G_{1}\right)$ and $L\left(G_{2}\right)$ are isomorphic. We also recall that an important modular sublattice of $L(G)$ is constituted by the normal subgroup lattice of $G$, usually denoted by $N(G)$.

The paper is organized as follows. In Section 2 we present some basic properties and results on the poset Iso $(G)$ associated to a finite group $G$. A complete description of this poset is given for several remarkable groups. Section 3 deals with the finite groups having the same poset of isomorphic subgroups. In the final section some conclusions and further research directions are indicated.

Most of our notation is standard and will usually not be repeated here. Basic definitions and results on lattices and groups can be found in [1, 5] and

[6, 17, 13], respectively. For subgroup lattice concepts we refer the reader to [11, 12, 14].

\section{The poset $\operatorname{Iso}(G)$}

Let $G$ be a finite group and $\operatorname{Iso}(G)$ be the set of equivalence classes of subgroups of $G$ with respect to the isomorphism relation, that is

$$
\operatorname{Iso}(G)=\{[H] \mid H \in L(G)\}, \text { where }[H]=\{K \in L(G) \mid K \cong H\} .
$$


Then it is easy to see that $\operatorname{Iso}(G)$ can be partially ordered by defining

$$
\left[H_{1}\right] \leq\left[H_{2}\right] \text { if and only if } K_{1} \subseteq K_{2} \text { for some } K_{1} \in\left[H_{1}\right] \text { and } K_{2} \in\left[H_{2}\right] \text {. }
$$

We remark that $\leq$ is weaker than the usual ordering relation on $C(G)$ and that the isomorphism relation is not a congruence on $L(G)$, even if in many cases the poset $(\operatorname{Iso}(G), \leq)$ becomes a lattice. We also must mention that the subposet of $\operatorname{Iso}(G)$ determined by all classes with a unique element is in fact the lattice $\operatorname{Sol}(G)$ of solitary subgroups of $G$, introduced and studied in [8].

First of all, we will look at the poset $\operatorname{Iso}(G)$ associated to some finite groups of small orders.

\section{Examples.}

1. Iso $\left(\mathbb{Z}_{p}\right)$ is a chain of length 1 , for any prime $p$.

2. $\operatorname{Iso}\left(\mathbb{Z}_{p} \times \mathbb{Z}_{p}\right) \cong \operatorname{Iso}\left(\mathbb{Z}_{p^{2}}\right)$ is a chain of length 2 , for any prime $p$.

3. $\operatorname{Iso}\left(\mathbb{Z}_{6}\right) \cong \operatorname{Iso}\left(S_{3}\right) \cong \operatorname{Iso}\left(D_{10}\right)$ is a direct product of two chains of length 2.

4. $\operatorname{Iso}\left(\mathbb{Z}_{2}^{3}\right) \cong \operatorname{Iso}\left(\mathbb{Z}_{8}\right) \cong \operatorname{Iso}\left(Q_{8}\right)$ is a chain of length 3 .

5. $\operatorname{Iso}\left(\mathbb{Z}_{2} \times \mathbb{Z}_{4}\right) \cong \operatorname{Iso}\left(D_{8}\right)$ is the lattice $\mathrm{C}_{5}$ (see page 5 of [11]).

6. $\operatorname{Iso}\left(A_{4}\right)$ is the pentagon lattice $\mathrm{E}_{5}$ (see page 5 of [11]).

It is well-known that a finite nilpotent group $G$ can be written as the direct product of its Sylow subgroups

$$
G=\chi_{i=1}^{k} G_{i},
$$

where $\left|G_{i}\right|=p_{i}^{\alpha_{i}}$, for all $i=1,2, \ldots, k$. Since the subgroups of a direct product of groups having coprime orders are also direct products (see Corollary of (4.19), [13], I), one obtains that

$$
L(G) \cong \chi_{i=1}^{k} L\left(G_{i}\right)
$$


This lattice direct decomposition is often used in order to reduce many problems on $L(G)$ to the subgroup lattices of finite $p$-groups. We easily observe that $(*)$ leads to

$$
\operatorname{Iso}(G) \cong{\underset{X}{X=1}}_{\text {Iso }}^{k}\left(G_{i}\right),
$$

that is the decomposability of $L(G)$ implies the decomposability of Iso $(G)$. Moreover, all posets $\operatorname{Iso}\left(G_{i}\right), i=1,2, \ldots, k$, are indecomposable, since each group $G_{i}$ possesses a unique class of isomorphism of subgroups of order $p_{i}$. The above example 3 shows that the converse implication fails: $\operatorname{Iso}\left(S_{3}\right)$ is decomposable, in contrast with $L\left(S_{3}\right)$.

In the following we shall focus on describing the poset Iso $(G)$ for several important classes of finite groups $G$. We start with abelian groups, for which we already know that the study is reduced to abelian $p$-groups.

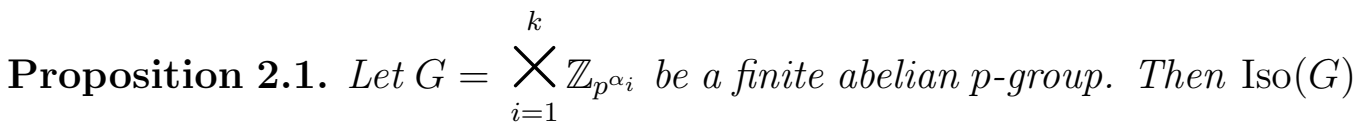
is an indecomposable distributive lattice and

$$
|\operatorname{Iso}(G)| \leq \sum_{i=0}^{\alpha} \pi(i),
$$

where $\alpha=\alpha_{1}+\alpha_{2}+\cdots+\alpha_{k}$ and $\pi(i)$ denotes the number of partitions of $i$, for all $i=0,1, \ldots, \alpha$.

Proof. Two subgroups of an arbitrary order $p^{m}$ of $G$ are isomorphic if and only if their types determine the same partition $\left(m_{1}, m_{2}, \ldots, m_{k}\right)$ of $m$ $\left(0 \leq m_{1} \leq m_{2} \leq \cdots \leq m_{k}\right)$. In this way, we can identify every class of isomorphic subgroups of $G$ with an element of the direct product

$$
C=\underset{i=1}{\stackrel{k}{X}} C_{\alpha_{i}},
$$

where $C_{\alpha_{i}}$ is the chain $0<1<2<\cdots<\alpha_{i}$, for all $i=1,2, \ldots, k$. Clearly, given $[H],[K] \in \operatorname{Iso}(G)\left(|H|=p^{m},|K|=p^{m^{\prime}}\right)$ that correspond to the partitions $\left(m_{1}, m_{2}, \ldots, m_{k}\right)$ and $\left(m_{1}^{\prime}, m_{2}^{\prime}, \ldots, m_{k}^{\prime}\right)$ of $m$ and $m^{\prime}$, respectively, we have

$$
\inf \{[H],[K]\}=[S] \text { and } \sup \{[H],[K]\}=[T] \text {, }
$$


where $[S]$ and $[T]$ are determined by the $k$-tuples $\left(\min \left\{m_{1}, m_{1}^{\prime}\right\}, \min \left\{m_{2}, m_{2}^{\prime}\right\}\right.$, $\left.\ldots, \min \left\{m_{k}, m_{k}^{\prime}\right\}\right)$ and $\left(\max \left\{m_{1}, m_{1}^{\prime}\right\}, \max \left\{m_{2}, m_{2}^{\prime}\right\}, \ldots, \max \left\{m_{k}, m_{k}^{\prime}\right\}\right)$.

Hence $\operatorname{Iso}(G)$ forms a lattice that can be embedded into a distributive lattice, namely $C$. This completes the proof.

Another remarkable class of finite groups for which a similar conclusion holds is constituted by the so-called ZM-groups, that is the finite groups all of whose Sylow subgroups are cyclic. It is well-known that two subgroups of such a group $G$ are conjugate if and only if they have the same order. Moreover, $C(G)$ is isomorphic to the lattice $L_{n}$ of all divisors of $n=|G|$ (see Theorem A of [4]). Thus we infer the following result.

Proposition 2.2. Let $G$ be a ZM-group of order $n$. Then the following lattice isomorphisms hold

$$
\operatorname{Iso}(G) \cong C(G) \cong L_{n}
$$

In particular, $\operatorname{Iso}(G)$ is a distributive lattice.

\section{Remarks.}

1. The conclusion of Proposition 2.2 is valid for the finite cyclic groups, which are in fact the simplest ZM-groups.

2. The lattice isomorphism from $\operatorname{Iso}(G)$ to $L_{n}$ in the above proposition is given by $[H] \mapsto|H|$, for all $[H] \in \operatorname{Iso}(G)$. For an arbitrary finite group $G$ of order $n$, this map is only isotone (that is, $[H] \leq[K]$ implies that $|H|$ divides $|K|)$.

3. There are finite groups $G$ such that $\operatorname{Iso}(G)$ is a lattice, but not a distributive or even a modular lattice. For example, in $\operatorname{Iso}\left(S_{2^{n}}\right), n \geq 4$, the classes determined by $S_{2^{n}}$, the three maximal subgroups of $S_{2^{n}}$ (that are isomorphic to $Q_{2^{n-1}}, D_{2^{n-1}}$ and $\mathbb{Z}_{2^{n-1}}$, respectively) and $\Phi\left(S_{2^{n}}\right)$ form a diamond and so $\operatorname{Iso}\left(S_{2^{n}}\right)$ is not distributive; on the other hand, we already have seen that $\operatorname{Iso}\left(A_{4}\right)$ is the pentagon lattice and therefore it is not modular.

Given a finite group $G$, some lattice-theoretical properties can be transferred from $\operatorname{Iso}(G)$ to $L(G)$. One of them is the complementation. 
Proposition 2.3. Let $G$ be a finite group. If $\operatorname{Iso}(G)$ is a complemented lattice, then $L(G)$ is also a complemented lattice.

Proof. Suppose that $\operatorname{Iso}(G)$ is a complemented lattice and denote by $\wedge^{\prime}$ and $\vee^{\prime}$ its binary operations. Then for every $[H] \in \operatorname{Iso}(G)$ there is $[K] \in \operatorname{Iso}(G)$ such that $[H] \wedge^{\prime}[K]=[1]$ and $[H] \vee^{\prime}[K]=[G]$. Since $H \wedge K$ is contained both in $H$ and $K$, we infer that $[H \wedge K] \leq[H],[K]$ and therefore $[H \wedge K] \leq$ $[H] \wedge^{\prime}[K]$. This implies $H \wedge K=1$. Similarly, one obtains $H \vee K=G$. Hence $K$ is a complement of $H$ in $L(G)$.

Remark. The converse of Proposition 2.3 is in general not true. For example, $H=\langle y\rangle$ has a complement in $L\left(D_{8}\right)$, namely $K=\left\langle x^{2}, x y\right\rangle$, but $[K]$ is not a complement of $[H]$ in $\operatorname{Iso}\left(D_{8}\right)$ (more precisely, $[H]$ has no complement in $\left.\operatorname{Iso}\left(D_{8}\right)\right)$.

Next, we will study the poset of classes of isomorphic subgroups for finite dihedral groups. Recall that the dihedral group $D_{2 n}(n \geq 2)$ is the symmetry group of a regular polygon with $n$ sides and it has the order $2 n$. The most convenient abstract description of $D_{2 n}$ is obtained by using its generators: a rotation $x$ of order $n$ and a reflection $y$ of order 2. Under these notations, we have

$$
D_{2 n}=\left\langle x, y \mid x^{n}=y^{2}=1, y x y=x^{-1}\right\rangle .
$$

It is well-known that for every divisor $r$ of $n, D_{2 n}$ possesses a subgroup isomorphic to $\mathbb{Z}_{r}$, namely $H_{0}^{r}=\left\langle x^{\frac{n}{r}}\right\rangle$, and $\frac{n}{r}$ subgroups isomorphic to $D_{2 r}$, namely $H_{i}^{r}=\left\langle x^{\frac{n}{r}}, x^{i-1} y\right\rangle, i=1,2, \ldots, \frac{n}{r}$. Then

$$
\left|L\left(D_{2 n}\right)\right|=\tau(n)+\sigma(n),
$$

where $\tau(n)$ and $\sigma(n)$ are the number and the sum of all divisors of $n$, respectively. We easily infer that

$$
\left|\operatorname{Iso}\left(D_{2 n}\right)\right|=\left\{\begin{array}{l}
2 \tau(n)-1, \text { for } n \text { odd } \\
2 \tau(n), \text { for } n \text { even. }
\end{array}\right.
$$

We are now able to determine the positive integers $n$ such that $\operatorname{Iso}\left(D_{2 n}\right)$ forms a lattice.

Proposition 2.4. The poset $\operatorname{Iso}\left(D_{2 n}\right)$ is a lattice if and only if either $n$ is odd or $n=2^{k}$ for some $k \in \mathbb{N}$. 
Proof. Suppose first that $n=p_{1}^{\alpha_{1}} p_{2}^{\alpha_{2}} \cdots p_{k}^{\alpha_{k}}$ with $p_{i}>2$ prime, $i=1,2, \ldots, k$, or $n=2^{k}$ for some $k \in \mathbb{N}$. Then, by using the above description of the subgroups of $D_{2 n}$, a standard induction argument on $k$ easily shows that $\operatorname{Iso}\left(D_{2 n}\right)$ forms a lattice.

Conversely, assume that $\operatorname{Iso}\left(D_{2 n}\right)$ is a lattice, but $n$ is of the form $n=$ $2^{\alpha} \beta$, where $\alpha \geq 1$ and $\beta \neq 1$ is odd. Then $D_{2 n}$ possesses the subgroups $H=\left\langle x^{2^{\alpha}}, y\right\rangle \cong D_{2 \beta}$ and $K=\left\langle x^{2^{\alpha-1}}\right\rangle \cong \mathbb{Z}_{2 \beta}$. Clearly, both $H$ and $K$ contain cyclic subgroups of orders 2 and $\beta$, which proves that $\left[C_{2}\right] \leq[H],[K]$ and $\left[C_{\beta}\right] \leq[H],[K]$ (here $C_{2}$ and $C_{\beta}$ are arbitrary cyclic subgroups of $D_{2 n}$ of orders 2 and $\beta$, respectively). It follows immediately that $\inf \{[H],[K]\}$ does not exist, a contradiction.

\section{Remarks.}

1. 12 is the smallest positive integer $n$ for which there is a finite group $G$ of order $n$ such that $\operatorname{Iso}(G)$ is not a lattice.

2. Another example of a group with the above property is the direct product $D_{8} \times \mathbb{Z}_{4}$. In this case the classes determined by the subgroups $H=\left\langle x^{2}\right\rangle \times \mathbb{Z}_{4}$ and $K=D_{8}$ does not possess an infimum, too.

Unfortunately, we failed in describing exhaustively the class of finite groups $G$ for which the poset $\operatorname{Iso}(G)$ is a (distributive/modular) lattice. We remark that such a group $G$ satisfies the following interesting property: "for every two distinct prime divisors $p$ and $q$ of $|G|$, either all subgroups of order $p q$ in $G$ are cyclic or all subgroups of order $p q$ in $G$ are non-abelian".

We end this section by characterizing all finite groups whose posets of classes of isomorphic subgroups are chains (i.e. distributive lattices of a very particular type).

Theorem 2.5. Let $G$ be a finite group. Then $\operatorname{Iso}(G)$ is a chain if and only if $G$ is either a cyclic p-group, an elementary abelian p-group, a non-abelian p-group of order $p^{3}$ and exponent $p$ or a quaternion group of order 8 .

Proof. It is clear that for a cyclic $p$-group, an elementary abelian $p$-group, a non-abelian $p$-group of order $p^{3}$ and exponent $p$ or a quaternion group of order 8, the poset of classes of isomorphic subgroups forms a chain.

Conversely, suppose that $\operatorname{Iso}(G)$ is a chain. Then $G$ is a $p$-group. Put $|G|=p^{n}$ and take a minimal normal subgroup $H$ of $G$. 
If $H$ is the unique subgroup of order $p$ of $G$, then (4.4) of [13], II, shows that $G$ is either cyclic or a generalized quaternion group $Q_{2^{n}}, n \geq 3$. It is well-known that the isomorphism classes of the maximal subgroups of $Q_{2^{n}}$ are $Q_{2^{n-1}}$ and $\mathbb{Z}_{2^{n-1}}$, and therefore Iso $\left(Q_{2^{n}}\right)$ is not a chain for $n \geq 4$. This holds only for $n=3$, that is for the quaternion group $Q_{8}$.

If $G$ possesses a minimal subgroup $K$ with $K \neq H$, then $H K$ is elementary abelian of order $p^{2}$. One obtains that there is no cyclic subgroup of order $p^{2}$ in $G$, in other words we have

$$
\exp (G)=p
$$

Obviously, if $G$ is abelian, then it is an elementary abelian $p$-group. In the following we will assume that $G$ is not abelian. Then $p$ is odd and $G$ contains a non-abelian subgroup of order $p^{3}$, say $N$ (more precisely, $N$ is isomorphic with the group $M\left(p^{3}\right)$, described in (4.13) of [13], II). Let $A$ be an abelian normal subgroup of maximal order of $G$ and set $|A|=p^{a}$. If $a \geq 3$ we infer that $A$ has a subgroup $A_{1}$ of order $p^{3}$. It follows that the classes $[N]$ and $\left[A_{1}\right]$ are not comparable, a contradiction. In this way, we have $a \leq 2$ and so $a \in\{1,2\}$. By Corollary 2, [13], I, page 94, we know that $2 n \leq a(a+1)$, which implies $n \leq 3$. This leads to $n=3$ and hence $G=N$ is a non-abelian $p$-group of order $p^{3}$ and exponent $p$, which completes the proof.

In particular, Theorem 2.5 shows that there are only two finite nonabelian groups $G$ with $\operatorname{Iso}(G)$ fully ordered, and each of them is of order $p^{3}$ for some prime $p$.

\section{$3 \quad$ Finite groups with the same poset of classes of isomorphic subgroups}

In this section we study when for two finite groups $G_{1}$ and $G_{2}$ the poset/lattice isomorphism $\operatorname{Iso}\left(G_{1}\right) \cong \operatorname{Iso}\left(G_{2}\right)$ holds. Obviously, a sufficient condition to have this isomorphism is $G_{1} \cong G_{2}$, but it is not necessary, as show the examples in Section 2. We also remark that the weaker condition $L\left(G_{1}\right) \cong L\left(G_{2}\right)$ does not imply that $\operatorname{Iso}\left(G_{1}\right) \cong \operatorname{Iso}\left(G_{2}\right)$ (for example, take $G_{1}=S_{3}$ and $\left.G_{2}=\mathbb{Z}_{3} \times \mathbb{Z}_{3}\right)$ and the same thing can be said about the converse implication.

We start with the following easy but important lemma. 
Lemma 3.1. Let $G_{1}$ be a finite p-group of order $p^{n}$. If $G_{2}$ is a finite group such that $\operatorname{Iso}\left(G_{1}\right) \cong \operatorname{Iso}\left(G_{2}\right)$, then $G_{2}$ is a q-group of order $q^{n}$.

Proof. The condition $\left|G_{1}\right|=p^{n}$ implies that Iso $\left(G_{1}\right)$ possesses a unique non-trivial element, namely the class determined by the subgroups of order $p$. Then $\operatorname{Iso}\left(G_{2}\right)$ satisfies a similar property, and so $G_{2}$ is a $q$-group for some prime $q$. One the other hand, we easily infer that all maximal chains of Iso $\left(G_{1}\right)$ are of length $n$. Since a poset isomorphism preserves the length of such a chain, one obtains that $\left|G_{2}\right|=q^{n}$, as desired.

The above lemma can be extended to finite groups of arbitrary orders in the following manner.

Theorem 3.2. Let $G_{1}$ and $G_{2}$ be two finite groups such that $\operatorname{Iso}\left(G_{1}\right) \cong$ $\operatorname{Iso}\left(G_{2}\right)$. If $\left|G_{1}\right|=p_{1}^{\alpha_{1}} p_{2}^{\alpha_{2}} \cdots p_{k}^{\alpha_{k}}$, where $p_{i}, i=1,2, \ldots, k$, are distinct primes, then we have $\left|G_{2}\right|=q_{1}^{\alpha_{1}} q_{2}^{\alpha_{2}} \cdots q_{k}^{\alpha_{k}}$ for some distinct primes $q_{1}, q_{2}, \ldots, q_{k}$.

Proof. Let $f: \operatorname{Iso}\left(G_{1}\right) \longrightarrow \operatorname{Iso}\left(G_{2}\right)$ be a poset isomorphism, $i \in\{1,2, \ldots, k\}$ and $S_{i}$ be a Sylow $p_{i}$-subgroup of $G_{1}$. If $f\left(\left[S_{i}\right]\right)=\left[S_{i}^{\prime}\right]$, then, by Lemma 3.1 , we have $\left|S_{i}^{\prime}\right|=q_{i}^{\alpha_{i}}$ for some prime $q_{i}$. Moreover, it is easy to see that $S_{i}^{\prime}$ is a Sylow subgroup of $G_{2}$. Hence $\left|G_{2}\right|$ is of the form $q_{1}^{\alpha_{1}} q_{2}^{\alpha_{2}} \cdots q_{k}^{\alpha_{k}}$ with $q_{1}, q_{2}, \ldots, q_{k}$ distinct primes, completing the proof.

Proposition 2.1 and Theorem 3.2 lead the following immediate characterization of the poset isomorphism $\operatorname{Iso}\left(G_{1}\right) \cong \operatorname{Iso}\left(G_{2}\right)$ for two finite abelian groups $G_{1}$ and $G_{2}$.

Corollary 3.3. Let $G_{1}$ and $G_{2}$ be two finite abelian groups of orders $p_{1}^{\alpha_{1}} p_{2}^{\alpha_{2}} \cdots p_{k}^{\alpha_{k}}$ and $q_{1}^{\beta_{1}} q_{2}^{\beta_{2}} \cdots q_{r}^{\beta_{r}}$, respectively. Then $\operatorname{Iso}\left(G_{1}\right) \cong \operatorname{Iso}\left(G_{2}\right)$ if and only if $k=r$ and there is a permutation $\sigma$ of $\{1,2, \ldots, k\}$ such that $\beta_{i}=\alpha_{\sigma(i)}$ and $\operatorname{Iso}\left(S_{q_{i}}^{\prime}\right) \cong$ Iso $\left(S_{p_{\sigma(i)}}\right)$, where $S_{q_{i}}^{\prime}$ is the Sylow $q_{i}$-subgroup of $G_{2}$ and $S_{p_{\sigma(i)}}$ is the Sylow $p_{\sigma(i)}$-subgroup of $G_{1}$, for all $i=1,2, \ldots, k$.

Example. By using Corollary 3.3, we easily infer that

$$
\operatorname{Iso}\left(\mathbb{Z}_{2} \times \mathbb{Z}_{6} \times \mathbb{Z}_{18}\right) \cong \operatorname{Iso}\left(\mathbb{Z}_{7} \times \mathbb{Z}_{6125}\right) .
$$

Lemma 3.1 shows that the class of finite $p$-groups is preserved by isomorphisms between their posets of classes of isomorphic subgroups. Other 
important classes of finite groups satisfying the same property are solvable groups, CLT-groups and supersolvable groups, respectively. This is due to the fact that if $f: \operatorname{Iso}\left(G_{1}\right) \longrightarrow \operatorname{Iso}\left(G_{2}\right)$ is a poset isomorphism and $G_{1}$ is of one of the above three types, then the strong connection between the orders of $G_{1}$ and $G_{2}$ assures the existence of Hall subgroups, of subgroups of any orders or the validity of the Jordan-Dedekind chain condition for $G_{2}$.

Corollary 3.4. The classes of finite solvable groups, CLT-groups and supersolvable groups are preserved by isomorphisms between their posets of classes of isomorphic subgroups.

We end this section by some results related to the uniqueness of a finite group with a given poset of classes of isomorphic subgroups.

Theorem 3.5. Let $n \geq 2$ be an integer which is not square-free. Then there are at least two non-isomorphic groups $G_{1}$ and $G_{2}$ of order $n$ such that $\operatorname{Iso}\left(G_{1}\right) \cong \operatorname{Iso}\left(G_{2}\right)$.

Proof. Let $n=p_{1}^{\alpha_{1}} p_{2}^{\alpha_{2}} \cdots p_{k}^{\alpha_{k}}$ be the decomposition of $n$ as a product of prime factors. We will proceed by induction on $k$.

Suppose first that $k=1$, that is $n=p_{1}^{\alpha_{1}}$ with $\alpha_{1} \geq 2$. For $\alpha_{1}=2$ we take $G_{1}=\mathbb{Z}_{p_{1}^{2}}$ and $G_{2}=\mathbb{Z}_{p_{1}} \times \mathbb{Z}_{p_{1}}$. For $\alpha_{1} \geq 3$ we take $G_{1}=M\left(p_{1}^{\alpha_{1}}\right)$ (see Theorem 4.1 of [13], II) and $G_{2}=\mathbb{Z}_{p_{1}^{\alpha_{1}-1}} \times \mathbb{Z}_{p_{1}}$ if $p_{1} \neq 2$, respectively $G_{1}=D_{p_{1}^{\alpha_{1}}}$ and $G_{2}=\mathbb{Z}_{p_{1}^{\alpha_{1}-1}} \times \mathbb{Z}_{p_{1}}$ if $p_{1}=2$.

Assume now that $k \geq 2$. By the inductive hypothesis, we can choose two non-isomorphic groups $H_{1}$ and $H_{2}$ of the order $p_{1}^{\alpha_{1}} p_{2}^{\alpha_{2}} \cdots p_{k-1}^{\alpha_{k-1}}$ such that $\operatorname{Iso}\left(H_{1}\right) \cong \operatorname{Iso}\left(H_{2}\right)$. Then it is a simple exercise to see that the groups $G_{1}=H_{1} \times \mathbb{Z}_{p_{k}^{\alpha_{k}}}$ and $G_{2}=H_{2} \times \mathbb{Z}_{p_{k}^{\alpha_{k}}}$ satisfy the desired conditions, completing the proof.

Inspired by Theorem 3.5, we came up with the following conjecture, which we have verified for several finite groups of small orders.

Conjecture. For every non-trivial finite group $G_{1}$ whose order is not squarefree there exists a finite group $G_{2}$ such that $\left|G_{1}\right|=\left|G_{2}\right|, \operatorname{Iso}\left(G_{1}\right) \cong \operatorname{Iso}\left(G_{2}\right)$ and $G_{1} \not G_{2}$.

Remark. The above conjecture says nothing else than the implication

$$
\left|G_{1}\right|=\left|G_{2}\right| \text { and } \operatorname{Iso}\left(G_{1}\right) \cong \operatorname{Iso}\left(G_{2}\right) \Longrightarrow G_{1} \cong G_{2}
$$

fails in all cases, except when $\left|G_{1}\right|=\left|G_{2}\right|$ is a square-free number. 


\section{Conclusions and further research}

All our previous results show that the poset consisting of all classes of isomorphic subgroups of a (finite) group can constitute a significant aspect of (finite) group theory. Clearly, the study started in this paper can successfully be extended to other classes of groups. It can be also generalized by studying the posets of isomorphic substructures of other algebraic structures (rings, modules, algebras, ... and so on). This will surely be the subject of some further research.

Finally, we mention several open problems concerning this topic.

Problem 4.1. Determine the finite groups $G$ for which the poset $\operatorname{Iso}(G)$ is a lattice and study the properties of this lattice.

Problem 4.2. What can be said about two arbitrary finite groups $G_{1}$ and $G_{2}$ satisfying $\operatorname{Iso}\left(G_{1}\right) \cong \operatorname{Iso}\left(G_{2}\right)$ ?

Problem 4.3. Given two finite groups $G_{1}$ and $G_{2}$, study the isomorphisms between the posets/lattices $\operatorname{Iso}\left(G_{1}\right)$ and $\operatorname{Iso}\left(G_{2}\right)$ induced by the isomorphisms or by the $L$-isomorphisms between $G_{1}$ and $G_{2}$.

Problem 4.4. The most natural generalization of the poset $\operatorname{Iso}(G)$ associated to a finite group $G$ is obtained by considering $L$-isomorphisms instead of group isomorphisms in its definition:

$$
\operatorname{Iso}^{\prime}(G)=\left\{[H]^{\prime} \mid H \in L(G)\right\} \text {, where }[H]^{\prime}=\{K \in L(G) \mid L(K) \cong L(H)\} \text {. }
$$

Investigate the above new poset $\operatorname{Iso}^{\prime}(G)$ with respect to the same ordering relation as for $\operatorname{Iso}(G)$.

Problem 4.5. Given a finite group $G$, study the posets of classes of subgroups with respect to other equivalence relations on $L(G)$ (or on other important subposets of $L(G))$. For example:

1. $H \sim_{1} K$ if and only if $|H|=|K|$;

2. $H \sim_{2} K$ if and only if there is $f \in \operatorname{Aut}(G)$ such that $f(H)=K$;

3. $H \sim_{3} K$ if and only if $\pi_{e}(H)=\pi_{e}(K)$ (that is, $H$ and $K$ have the same set of element orders). 
Problem 4.6. The concept of solitary quotient of a finite group has been defined in [15] as the dual concept of solitary subgroup. Following the same technique, we can construct a "dual" for the set Iso $(G)$, namely

$$
\operatorname{QIso}(G)=\{[H] \mid H \in N(G)\} \text {, where }[H]=\{K \in N(G) \mid G / K \cong G / H\} \text {. }
$$

Endow this set with a suitable ordering relation and study some similar problems.

Acknowledgements. The author is grateful to the reviewer for its remarks which improve the previous version of the paper.

\section{References}

[1] Birkhoff, G., Lattice theory, Amer. Math. Soc., Providence, R.I., 1967.

[2] Brandl, R., Posets of subgroups in p-groups, Comm. Algebra 20 (1992), 3043-3054.

[3] Brandl, R., Verardi, L., Metacyclic p-groups and their conjugacy classes of subgroups, Glasgow Math. J. 35 (1993), 339-344.

[4] Brandl, R., Cutolo, G., Rinauro, S., Posets of subgroups of groups and distributivity, Boll. U.M.I. 9-A (1995), 217-223.

[5] Grätzer, G., General lattice theory, Academic Press, New York, 1978.

[6] Huppert, B., Endliche Gruppen, I, Springer Verlag, Berlin, 1967.

[7] Isaacs, I.M., Finite group theory, Amer. Math. Soc., Providence, R.I., 2008.

[8] Kaplan, G., Levy, D., Solitary subgroups, Comm. Algebra 37 (2009), 1873-1883.

[9] Mainardis, M., On the poset of conjugacy classes of subgroups of finite p-groups, Comm. Algebra 25 (1997), 3155-3177.

[10] Mainardis, M., Finite groups whose poset of conjugacy classes of subgroups is isomorphic to the one of an abelian group, Boll. U.M.I. 1-B (1998), 691-698. 
[11] Schmidt, R., Subgroup lattices of groups, de Gruyter Expositions in Mathematics 14, de Gruyter, Berlin, 1994.

[12] Suzuki, M., Structure of a group and the structure of its lattice of subgroups, Ergebnisse der Mathematik und ihrer Grenzgebiete, Neue Folge, Heft 10, Springer Verlag, Berlin-Göttingen-Heidelberg, 1956.

[13] Suzuki, M., Group theory, I, II, Springer Verlag, Berlin, 1982, 1986.

[14] Tărnăuceanu, M., Groups determined by posets of subgroups, Ed. Matrix Rom, Bucureşti, 2006.

[15] Tărnăuceanu, M., Solitary quotients of finite groups, Cent. Eur. J. Math. 10 (2012), 740-747, doi: 10.2478/s11533-012-0003-0.

\author{
Marius Tărnăuceanu \\ Faculty of Mathematics \\ "Al.I. Cuza" University \\ Iaşi, Romania \\ e-mail: tarnauc@uaic.ro
}

